\title{
Lytic transglycosylases: Bacterial space-making autolysins
}

\author{
Edie Scheurwater, Chris W. Reid ${ }^{1}$, Anthony J. Clarke* \\ Department of Molecular and Cellular Biology, University of Guelph, \\ Guelph, Ontario N1G 2W1, Canada
}

Received 13 March 2007; received in revised form 20 March 2007; accepted 21 March 2007

Available online 30 March 2007

\begin{abstract}
Lytic transglycosylases are an important class of bacterial enzymes that act on peptidoglycan with the same substrate specificity as lysozyme. Unlike the latter enzymes, however, the lytic transglycosylases are not hydrolases but instead cleave the glycosidic linkage between $\mathrm{N}$-actetylmuramoyl and $\mathrm{N}$-acetylglucosaminyl residues with the concomitant formation of a 1,6-anydromuramoyl product. They are ubiquitous in bacteria which produce a compliment of different forms that are responsible for creating space within the peptidoglycan sacculus for its biosynthesis and recycling, cell division, and the insertion of cell-envelope spanning structures, such as flagella and secretion systems. As well, the lytic transglyosylases may have a role in pathogenesis of some bacterial species. Given their important role in bacterial cell wall metabolism, the lytic transglycosylases may present an attractive new target for the development of broad-spectrum antibiotics.
\end{abstract}

(C) 2007 Elsevier Ltd. All rights reserved.

Keywords: Lytic transglycosylases; Peptidoglycan; Catalytic mechanism; Bacterial cell walls

\section{Introduction}

The bacterial cell wall component peptidoglycan (PG; synonym, murein) is composed of glycan chains of alternating $\mathrm{N}$-acetylglucosamine (GlcNAc) and $\mathrm{N}$ acetylmuramic acid (MurNAc) that are cross-linked by peptides associated with the lactyl moiety of MurNAc. This heteropolymer imparts a mesh-like sacculus that surrounds the bacterial cell conferring strength, sup-

Abbreviations: LTs, lytic transglycosylases; PG, peptidoglycan; PBP, pencillin-binding protein; GlcNAc, $N$-acetylglucosamine; MurNAc, $N$-acetylmuramic acid

* Corresponding author. Tel.: +1 519824 4120;

fax: +1 5198371802

E-mail address: aclarke@uoguelph.ca (A.J. Clarke).

${ }^{1}$ Present address: Institute for Biological Sciences, National Research Council of Canada, 100 Sussex Drive, Ottawa, Ontario K1A 0R6, Canada. port, and shape, as well as resistance to internal turgor pressures. Maintaining the integrity of the PG sacculus is vital to cell viability and its importance is reflected by the number of different classes of antibiotics that target PG biosynthesis, including the glycopeptide vancomycin and the $\beta$-lactams. However, the PG sacculus is not a static structure, rather it is continually expanded and turned over. This metabolism also involves the creation of sites for the insertion of flagella and the creation of pores for secretion systems. A key class of enzymes responsible for cleaving PG to accommodate these requirements are the lytic transglycosylases (LTs). The LTs lyse PG with the same substrate specificity as the lysozymes, viz. the $\beta-1,4$ glycosydic bond between MurNAc and GlcNAc. However, unlike the lysozymes, the LTs are not hydrolases but instead cleave PG with concomitant formation of an intramolecular 1,6anhydromuramoyl reaction product (Höltje, Mirelman, Sharon, \& Schwarz, 1975) (Fig. 1). 
(A)

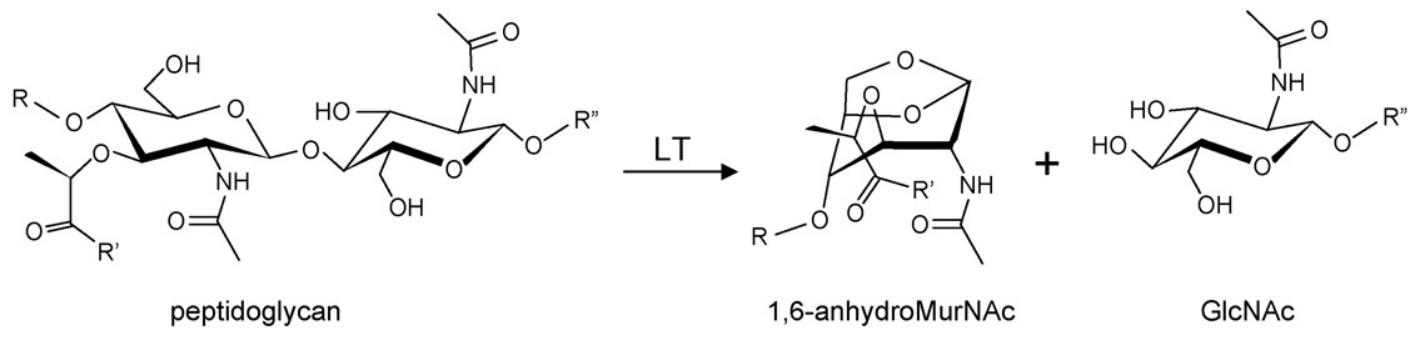

(B)

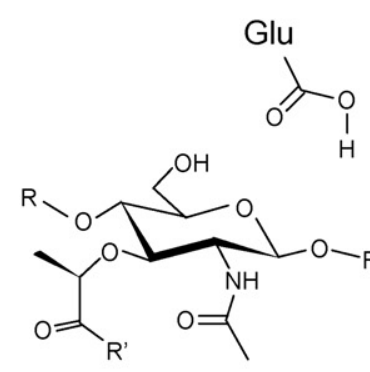

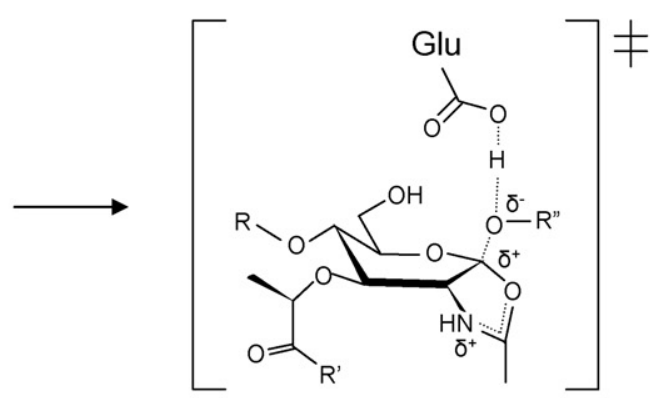
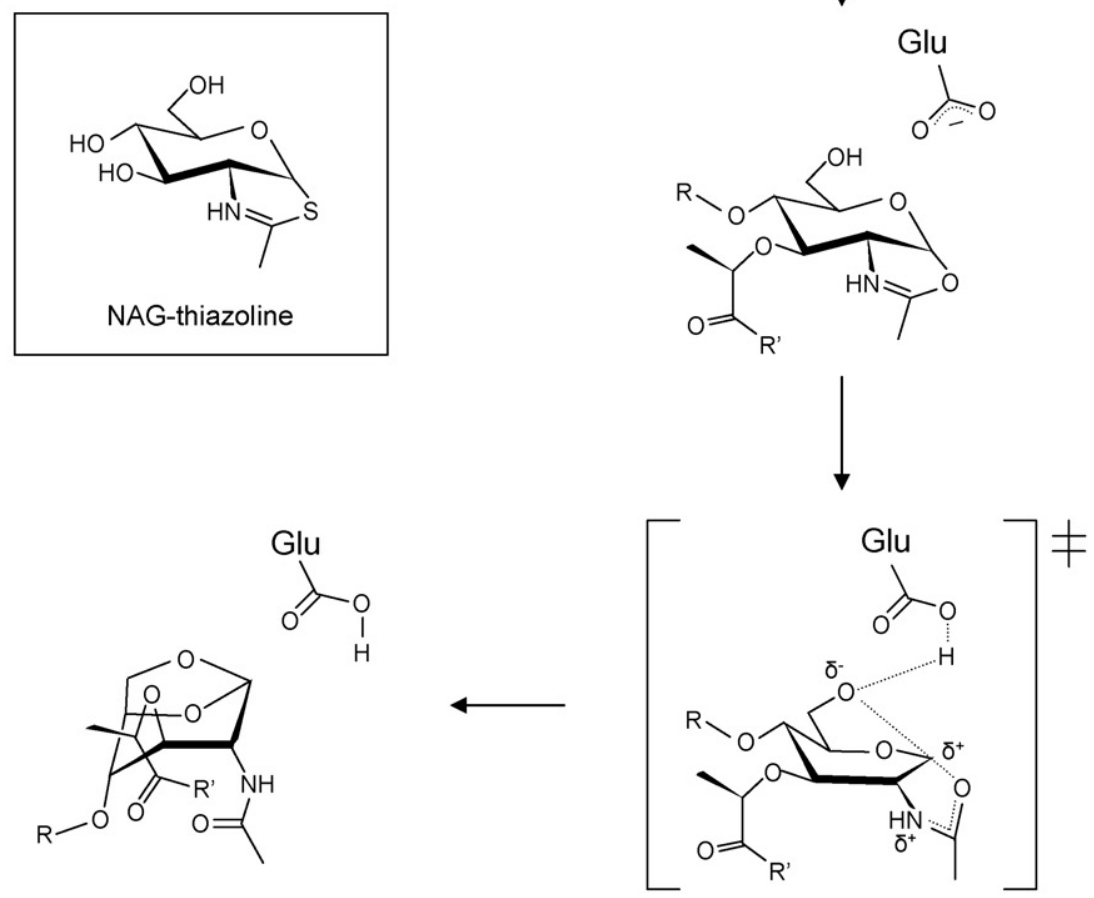

Fig. 1. Reaction and proposed mechanism catalyzed by LTs. (A) The LTs catalyze the cleavage of peptidoglycan between MurNAc and GlcNAc residues with the formation of 1,6-anhydromuramoyl residues. (B) Proposed reaction mechanism of LTs. The catalytic Glu serves initially as an acid to protonate the glycosidic linkage to be cleaved leading to the formation of a muramoyl oxazolinium-ion intermediate, and then as a base to abstract the C-6 hydroxyl proton of the oxazolinium ion promoting its collapse and concomitant formation of the 1,6-anhydromuramoyl product. Inset: Structure of NAG-thiazoline, an analog of the proposed muramoyl oxazolinium-ion reaction intermediate. 


\section{Families of LTs}

LTs are ubiquitous amongst the eubacteria except for the mycoplasmas. A variety of LTs exist and a classification scheme has been developed that involves four distinct families based on sequence similarities and identified consensus motifs (Blackburn \& Clarke, 2001) (Fig. 2). Of these, family 1 is a superfamily of five subfamilies with each sharing some limited sequence similarity to the goose-type lysozymes. With the plethora of sequence information now available, a more recent analysis suggests that family 3 also could be further divided into subfamilies (Reid, Blackburn, \& Clarke, 2006). Family 4 enzymes are primarily of bacteriophage origin.
Bacteria are found to encode a compliment of the different LTs from the various families. For example, $E$. coli is currently known to produce six LTs, one from each of families 1A (Slt70), 1C (MltC), 1D (MltD), 1E (EmtA), 2 (MltA) and 3 (MltB). In contrast, the opportunistic pathogen Pseudomonas aeruginosa does not appear to encode family $1 \mathrm{C}$ and $1 \mathrm{E}$ enzymes, but instead produces four MltB isozymes in addition to the other LTs produced by Escherichia coli (Blackburn \& Clarke, 2001). This apparent redundancy is analogous to the situation with the production of a number and variety of penicillin-binding proteins (PBPs) that are responsible for the final stages of PG biosynthesis. Also like the PBPs, "functional equivalency" amongst the LTs appears to permit the
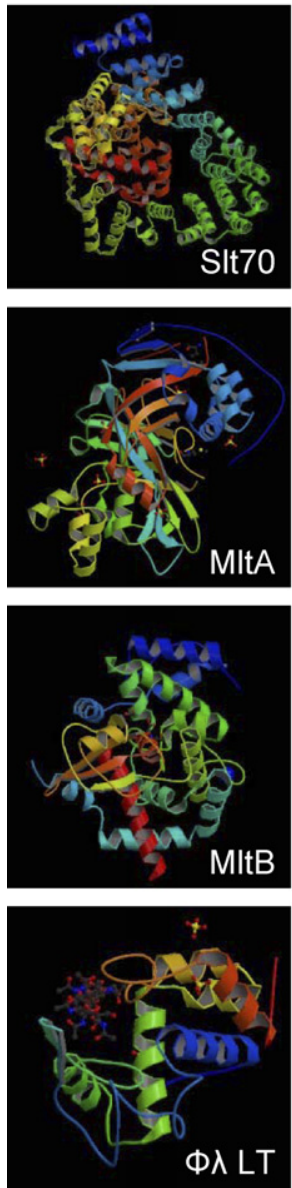

\section{Family}

$1 \mathrm{~A}$
$1 \mathrm{~B}$
$1 \mathrm{C}$
$1 \mathrm{D}$
$1 \mathrm{E}$

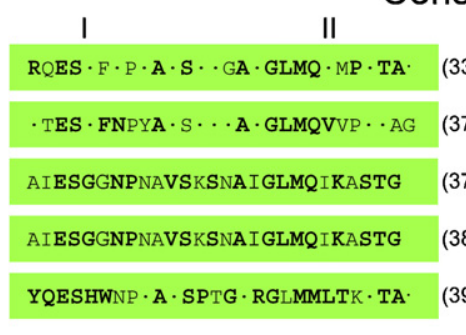

Consensus Motifs

\section{III}
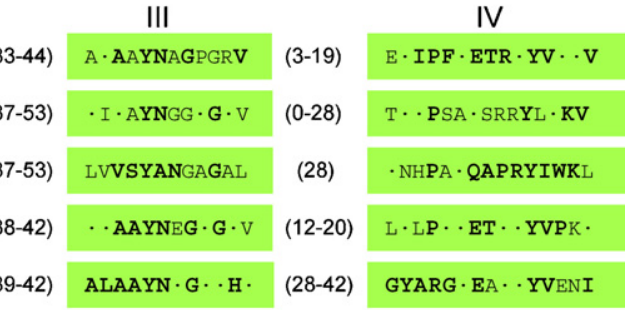

2

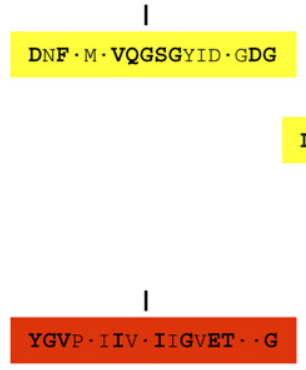

3

$(9-10)$

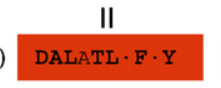

$(30-36)$

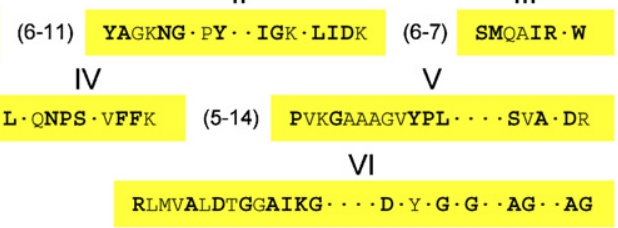

$(3-17)$

(23-31)

(2)

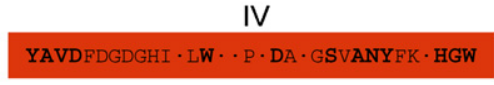

III

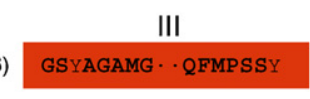

IV

II

$\begin{array}{llll}\text { A } \cdots \cdot \text { SEG } & (6-12) & \text { YDVIVGGELFTDYSDHPRKLV }\end{array}$

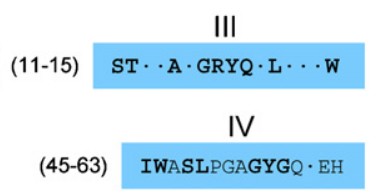

Fig. 2. Structure of LTs. The three-dimensional structures of LTs representing the four families are depicted beside their defining consensus motifs. The structures presented are of: E. coli Slt70 (family 1A; PDB, 1QTE), N. gonorrhoeae MltA (family 2; PDB, 2G6G), E. coli MltB (family 3; PDB, 1QUS), and bacteriophage lambda LT (family 4; PDB, 1D9U). Residues in boldface type of the consensus motifs (identified by roman numerals) are present in greater than $80 \%$ of the sequences of the individual families whereas those in small type are present in at least $50 \%$ of the sequences. The numbers in parentheses denote the number of residues between the motifs. 
elimination of one or more without eliciting a lethal effect, at least under in vitro conditions (Blackburn \& Clarke, 2002; Heidrich, Ursinus, Berger, Schwarz, Höltje, 2002).

\section{Structure and function relationship of LTs}

\subsection{Three-dimensional structures}

Crystal structures have been solved for five LTs, representing families $1 \mathrm{~A}$ (E. coli Slt70, Thunnissen, Isaacs, \& Dijkstra, 1995), 2 (E. coli MltA, van Straaten, Dijkstra, Vollmer, \& Thunnissen, 2005; Neisseria gonorrhoeae MltA, Powell, Liu, Nicholas, \& Davies, 2006), 3 (E. coli Slt35, a naturally occurring product of MltB, van Asselt, Dijkstra, et al., 1999), and 4 (bacteriophage lambda LT, Leung, Duewel, Honek, \& Berghuis, 2001) (Fig. 2). Despite lacking sequence similarity and having disparity in overall tertiary structure, the family $1 \mathrm{~A}, 3$ and 4 LTs are mostly $\alpha$-helical and their catalytic domains appear to possess a "lysozyme" fold. In contrast, MltA (family 2) is uniquely structured as a beta-barrel that is more reminiscent of an endoglucanase $\mathrm{V}$ fold. Notwithstanding these differences in catalytic folds, the respective active site clefts of Slt70, MltA and MltB are comprised of four substrate binding subsites (labeled $-2,-1,+1$, and $+2)$ that can accommodate the aminosugar residues of a tetrasaccharide substrate (Powell et al., 2006; Thunnissen, Rozeboom, Kalk, \& Dijkstra, 1995; van Asselt, Kalk, \& Dijkstra, 2000; van Asselt, Thunnissen, \& Dijkstra, 1999; van Straaten et al., 2005). This subsite architecture is consistent with the exo-activity of these LTs, as they catalyze the release of disaccharide anhydromuropeptides from either the reducing or non-reducing ends of PG. The endo-acting bacteriophage LTs of family 4 accommodate hexasaccharide substrates within their active site clefts (Leung et al., 2001).

The MurNAc and GlcNAc residues of a PG metabolite are seen to bind the -1 and +1 subsites, respectively, accounting for the reaction specificity of LT function. Protein engineering and kinetic studies with family 3 LTs from $P$. aeruginosa has led to the identification of important structural requirements of both binding ligands and amino acid residues on the enzymes for substrate binding. In particular, extensive interactions between amino acid residues comprising subsite -1 and both the lactyl moiety of bound MurNAc and its associated peptide are critical for the proper orientation of substrate for lytic activity (Reid et al., 2006; Reid, Brewer, \& Clarke, 2004).

\subsection{Mechanism of action}

A single acidic residue appears to be positioned at the center of the active site clefts of each LT between subsites +1 and -1 irrespective of its overall length. This residue is thought to function as the catalytic acid/base for bond cleavage by a mechanism involving substrate-assisted catalysis (Reid, Blackburn, Legaree, Auzanneau, \& Clarke, 2004; Thunnissen et al., 1994; van Asselt, Dijkstra, et al., 1999). Thus, the catalytic residue would act initially as a general acid, donating its proton to the glycosydic oxygen of the scissile bond (Fig. 1). As bond cleavage occurs, the putative oxocarbenium ion transition state would be stabilized through the formation of an oxazolinium intermediate involving the $\mathrm{N}$-acetyl group of the muramoyl residue at subsite 1 . The deprotonated catalytic residue then acts as a general base to abstract a proton from the C6-OH of MurNAc thereby allowing for an intramolecular nucleophilic attack at $\mathrm{C} 1$ collapsing the oxazoline intermediate with the concomitant formation of the 1,6-anhydro reaction product. Experimental evidence for this proposed reaction mechanism has been obtained from inhibition studies using NAG-thiazoline (Fig. 2), an analog of the putative oxazolinium intermediate (Reid, Blackburn, et al., 2004).

\section{Biological functions}

LTs can be viewed as space-making enzymes; they cleave glycosydic bonds within the PG sacculus to allow for a number of different processes to occur. They are critical for the expansion of the sacculus and consequently cell growth by creating sites for the insertion of PG precursors (reviewed in Höltje, 1998). They are also required for PG turnover and, in Gram-negative bacteria, for recycling where released 1,6-anhydromuropeptides are transported back into the cytoplasm for reuse, and in higher concentrations, induce $\beta$-lactamase production. Together with amidases, LTs function to split the septum thereby permitting the separation of dividing cells (Heidrich et al., 2002). They have also been implicated with endospore germination and facilitating the insertion of protein complexes that extend through the PG sacculus, such as secretion systems, flagella, and pili (reviewed in Koraimann, 2003). Such systems are too large to pass through the natural pores within the sacculus and would therefore require the action of a PG cleaving enzyme to locally remodel the layer and form a large enough space for the system.

Given the assorted, specific functions ascribed to the LTs, it is tempting to correlate their family relationship with function. The limited information currently 
available suggests that family 1 LTs are involved with conjugation and secretion systems (VirB, Agrobacterium tumefaciens; IpgF, Shegella flexneri), family 2 with septation (LtgC, N. gonorrhoeae; GNA33, N. meningitidis), family 3 with flagella and pili formation, and sporulation (PleA, Caulobacter cresentus; $\mathrm{BfpH}$, E. coli EPEC; SleB Bacillus subtilis), and family 4 with bacteriophageinduced lysis.

LTs have been suggested to contribute to pathogenesis (reviewed in Cloud-Hansen et al., 2006). A number of LTs from different animal and plant pathogens, including Haemophilus influenza, N. meningitidis, S. flexneri, Erwinia amylovara, and Pseudomonas syringae have been shown to be up-regulated during host infection. As well, LT reaction products have been demonstrated to play a role in infections. PG fragments in general elicit many of the general symptoms of bacterial infections such as fever (pyrogenicity), lack of appetite, and sleepiness (somnogenicity) and stimulate the NOD1 and NOD2 intracellular receptors. In addition to these, the LT product GlcNAc-anhMurNAC-tetrapeptide serves as the cytotoxin responsible for the pathology of both Bordetella pertussis and $N$. gonorrhoeae infections. As many bacteria release this PG metabolite, it is likely that other pathobiological roles will eventually be ascribed to it.

\section{Control of LT activity}

LTs are considered to be autolytic due to their ability to cause complete cellular lysis if their activity is allowed to proceed uncontrolled. Given this and the fact that a bacterium produces a number of different LTs, it is crucial for the cell to control LT action. While little in this regard is known, one approach appears to involve chemical modification of substrate. For example, $O$-acetylation of PG occurs at the C-6 hydroxyl group of muramoyl residues thereby precluding the formation of the 1,6anhydromuramoyl product of LT activity (Blackburn \& Clarke, 2002).

Physical association and separation presents a second level of control of LT activity. In $E$. coli and $P$. aeruginosa, most LTs are peripheral membrane-bound lipoproteins associated with the inner (periplasmic) leaflet of the outer membrane (Blackburn \& Clarke, 2002; Höltje, 1998). In addition, both these and the naturally soluble LTs appear to form complexes with the PBPs which are also peripheral membrane proteins but associated with the outer leaflet of the cytoplasmic membrane (Koraimann, 2003). Hence, these protein complexes serve to fuse the two membranes together and sandwich the PG sacculus between them. Such associa- tions and physical constraints would guarantee that lysis is coupled with the biosynthetic transglycosylase activity of the PBPs to either extend the cell length or form division sites. LTs have also been found to be associated with extensive protein complexes that comprise secretion systems (e.g. Höppner, Carle, Sivanesan, Hoeppner, $\&$ Baron, 2005). For these, only limited lytic activity would be required to permit the extrusion of the complete secretion complex of proteins through the PG layer. The physical association of the LT responsible with the secretion apparatus would permit the appropriate coordination of this required lytic activity while precluding catastrophic autolysis.

\section{New bacterial target?}

Bacterial resistance to antibiotics is a major concern and the search for new antibiotic targets is imperative. LTs would appear to present an interesting potential new target. They are critical to bacterial cell function and reproduction, and they act on a structure unique to bacteria. Moreover, unlike the situation with a number of important antibacterials (e.g. $\beta$-lactams and vancomycin) that target the metabolism of the stem peptide on PG where variability may occur, the LTs function at a site that is both unique to and invariant in PG, viz. its muramoyl residues. Such consistency of the substrate would suggest that evolution of resistance to inhibitors would be slow to develop. Preliminary studies with the inhibitor NAG-thizaoline have shown that targeting the LTs does indeed lead to significant changes in the physical properties and morphology of bacterial cell walls, despite the poor affinity of the compound for the enzyme (Reid, Blackburn, \& Clarke, 2004). Thus, these promising results provide an impetus for further work toward the design more specific mechanism-based inhibitors that may prove useful in our constant struggle with bacterial pathogens.

\section{References}

Blackburn, N. T., \& Clarke, A. J. (2001). Identification of four families of peptidoglycan lytic transglycosylases. J. Mol. Evol., 52, 78-84.

Blackburn, N. T., \& Clarke, A. J. (2002). Characterization of soluble and membrane-bound family 3 lytic transglycosylases from Pseudomonas aeruginosa. Biochemistry, 41, 1001-1013.

Cloud-Hansen, K. A., Peterson, S. B., Stabb, E. V., Goldman, W. E., McFall-Ngai, M. J., \& Handelsman, J. (2006). Breaching the great wall: peptidoglycan and microbial interactions. Nat. Rev. Microbiol., 4, 710-716.

Heidrich, C., Ursinus, A., Berger, J., Schwarz, H., \& Höltje, J.-V. (2002). Effects of multiple deletions of murein hydrolases on viability, septum cleavage, and sensitivity to large toxic molecules in Escherichia coli. J. Bacteriol., 184, 6093-6099. 
Höltje, J.-V. (1998). Growth of the stress-bearing and shapemaintaining murein sacculus of Escherichia coli. Microbiol. Mol. Biol. Rev., 62, 181-203.

Höltje, J.-V., Mirelman, D., Sharon, N., \& Schwarz, U. (1975). Novel type of murein transglycosylase in Escherichia coli. J. Bacteriol., 124, 1067-1076.

Höppner, C., Carle, A., Sivanesan, D., Hoeppner, S., \& Baron, C. (2005). The putative lytic transglycosylase VirB1 from Brucella suis interacts with the type IV secretion system core components VirB8, VirB9 and VirB11. Microbiology, 151, 3469-3482.

Koraimann, G. (2003). Lytic transglycosylases in macromolecular transport systems of Gram-negative bacteria. Cell. Mol. Life Sci., 60, 2371-2388.

Leung, A. K.-W., Duewel, H. S., Honek, J. F., \& Berghuis, A. M. (2001). Crystal structure of the lytic transglycosylase from bacteriophage lambda in complex with hexa- $N$-acetylchitohexaose. Biochemistry, 40, 5665-5673.

Powell, A. J., Liu, Z. J., Nicholas, R. A., \& Davies, C. (2006). Crystal structures of the lytic transglycosylase MltA from $N$. gonorrhoeae and E. coli: insights into interdomain movements and substrate binding. J. Mol. Biol., 359, 122-136.

Reid, C. W., Blackburn, N. T., \& Clarke, A. J. (2004). The effect of NAG-thiazoline on morphology and surface hydrophobicity of Escherichia coli. FEMS Microbiol. Lett., 234, 343-348.

Reid, C. W., Blackburn, N. T., \& Clarke, A. J. (2006). Role of arginine residues in the active site of the membrane-bound lytic transglycosylase B from Pseudomonas aeruginosa. Biochemistry, 45, 2129-2138.

Reid, C. W., Blackburn, N. T., Legaree, B. A., Auzanneau, F. I., \& Clarke, A. J. (2004). Inhibition of membrane-bound lytic transglycosylase B by NAG-thiazoline. FEBS Lett., 574, 73-79.
Reid, C. W., Brewer, D., \& Clarke, A. J. (2004). Substrate binding affinity of Pseudomonas aeruginosa membrane-bound lytic transglycosylase B by hydrogen-deuterium exchange MALDI MS. Biochemistry, 43, 11275-11282.

Thunnissen, A.-M. W. H., Dijkstra, A. J., Kalk, K. H., Rozeboom, H. J., Engel, H., Keck, W., et al. (1994). Doughnut-shaped structure of a bacterial muramidase revealed by X-ray crystallography. Nature, 367, 750-753.

Thunnissen, A.-M. W. H., Isaacs, N. W., \& Dijkstra, B. W. (1995). The catalytic domain of a bacterial lytic transglycosylase defines a novel class of lysozymes. Proteins, 22, 245-258.

Thunnissen, A.-M. W. H., Rozeboom, H. J., Kalk, K. H., \& Dijkstra, B. W. (1995). Structure of the 70-kDa soluble lytic transglycosylase complexed with bulgecin A. Implications for the enzymatic mechanism. Biochemistry, 34, 12729-12737.

van Asselt, E. J., Dijkstra, A. J., Kalk, K. H., Takacs, B., Keck, W., \& Dijkstra, B. W. (1999). Crystal structure of Escherichia coli lytic transglycosylase Slt35 reveals a lysozyme-like catalytic domain with an EF-hand. Structure, 7, 1167-1180.

van Asselt, E. J., Kalk, K. H., \& Dijkstra, B. W. (2000). Crystallographic studies of the interactions of Escherichia coli lytic transglycosylase S1t35 with peptidoglycan. Biochemistry, 39, 1924-1934.

van Asselt, E. J., Thunnissen, A.-M. W. H., \& Dijkstra, B. W. (1999). High resolution crystal structures of the Escherichia coli lytic transglycosylase Slt70 and its complex with a peptidoglycan fragment. J. Mol. Biol., 291, 877-898.

van Straaten, K. E., Dijkstra, B. W., Vollmer, W., \& Thunnissen, A.M. W. H. (2005). Crystal structure of MltA from Escherichia coli reveals a unique lytic transglycosylase fold. J. Mol. Biol., 352, 1068-1082. 